\title{
A CLASS OF NORMAL DILATION MATRICES AFFIRMING THE MARCUS-DE OLIVEIRA CONJECTURE
}

\author{
KiJTi RodTES
}

Abstract. In this article, we provide a class of normal dilation matrices affirming the Marcus-de Oliveira conjecture.

Mathematics subject classification (2010): 15A15, 15A60, 15A86.

Keywords and phrases: Normal dilation, normal matrices, Marcus de Oliveira conjecture.

\section{REFERENCES}

[1] N. Bebiano, A. Kovacec, And J. DA Providencia, The validity of the Marcus-de Oliveira conjecture for essentially Hermitian matrices, Second Conference of the International Linear Algebra Society (ILAS) (Lisbon, 1992), Linear Algebra Appl., 197/198: 411-427, 1994.

[2] N. Bebiano, J. K. Merikoski, And J. Da Providencia, On a conjecture of G. N. de Oliveira on determinants, Linear and Multilinear Algebra 20: 167-170, 1987.

[3] G. N. DE OliveirA, Normal matrices (research problem), Linear and Multilinear Algebra 12: 153$154,1982$.

[4] S. W. Drury, On Symmetric Functions of the Eigenvalues of the Sum of Two Hermitian Matrices, Linear Algebra Appl. 176: 211-222, 1992.

[5] S. W. DRURY, Essentially Hermitian matrices revisited, Electron. J. Linear Algebra 15: 285-296, 2006.

[6] S. W. DRURY, OMC for scalar multiples of unitaries, Linear Algebra Appl. 422 (1): 318-325, 2007.

[7] S. W. DRURY AND B. ClOAD, On the determinantal conjecture of Marcus and de Oliveira, Linear Algebra Appl. 177: 105-109, 1992.

[8] M. FIEDLER, Bounds for the determinant of the sum of hermitian matrices, Proc. Amer. Math. Soc. 30: 27-31, 1971.

[9] M. Marcus, Derivations, Plucker relations, and the numerical range, Indiana Univ. Math. J. 22: $1137-1149,1972 / 73$.

[10] J. K. MERIKOS Ki AND A. VirTAnen, Some notes on de Oliveira's determinantal conjecture, Linear Algebra Appl. 121: 345-352, 1989.

[11] X. ZHAN, Open problems in matrix theory, ICCM, II. 1-4, 2007. 